\title{
Erratum: "Hydrodynamic Mechanism of Bleaching a Strongly Absorbing Liquid by a Laser Pulse" [Doklady Physics 48 (2), 90 (2003); Doklady Akademii Nauk 388, 5 (2003)]
}

\section{A. N. Kucherov}

In my paper "Hydrodynamic Mechanism of Bleaching a Strongly Absorbing Liquid by a Laser Pulse," formula (2) should be read as

$$
T_{\text {trans }}=\iint_{0}^{\infty} f\left(\frac{t}{\tau}\right) \exp \left[-\frac{\alpha \rho(r, t) \Delta z}{\rho_{0}}-\left(\frac{r}{r_{0}}\right)^{2}\right] \frac{2 r d r}{r_{0}^{2}} \frac{d t}{\tau}
$$

Translated by G. Merzon 